\title{
BATCH ROUNDNESS CHARACTERIZATION AND EVALUATION
}

\author{
Qimi Jiang, Hsi-Yung Feng \\ Department of Mechanical and Materials Engineering \\ The University of Western Ontario \\ London, Ontario, Canada N6A $5 B 9$ \\ qimi.jiang.1@ulaval.ca,sfeng@eng.uwo.ca
}

\begin{abstract}
A new concept referred to as the batch roundness is presented in this paper. This concept is derived to illustrate the worst possible form error for a batch of circular features machined under the same conditions. It is to be used as a statistical quality measure for such a batch of circular features and evaluated by analyzing their systematic and random form error components. The definition of batch roundness is introduced first and the associated evaluation algorithm is then presented. The evaltuation algorithm starts by characterizing the deterministic profile for the batch of circular features. When the deterministic profile is obtained, the residuals, which are regarded as the random form error component, are available. The batch roundness can then be evaluated and the corresponding confidence level of the batch roundness zone determined. Case studies using both the simulated and experimental data sets have successfully demonstrated that the batch roundness can be reliably estimated from the inspection data of only one circular feature in the batch. This unique feature of the presented algorithm will hold as long as the measurement data size is adequate and the relative magnitude of the random form error component with respect to the batch roundness is not too large.
\end{abstract}

\section{INTRODUCTION}

In today's manufacturing industry, discrete parts are often produced in batch under nearly the same conditions. This motivates many research studies that have focused on the related economic or financial issues such as the calculation of production costs (Lee and Leonard, 1992; Primrose, 1993; Shiu, 1991; Koziarski and Królikowski, 1998), the performance analysis of batch production systems (Garavelli, 2001), and batch production scheduling (Heywood et al., 1997). If a batch of mechanical parts with circular features is machined under the same conditions, their form errors (or roundness) should contain very similar systematic and random components. This makes it possible to estimate the worst possible roundness value for parts in the batch based on the circular measurement data from only one of the parts. Since such a roundness value describes the overall quality for a batch of manufactured circular features, it is named here as the batch roundness. 
This batch roundness value is very important to the successful implementation of modern monitoring, diagnostic and control technologies in production. It can be used as a quality measure for conformance checking of a batch of manufactured parts and more importantly, as a feedback input for proper corrective actions to the associated manufacturing processes to improve overall quality of the batch.

According to the ASME Y14.5M-1994 standard, the form error for a circular feature (roundness) is the gap between two concentric circles that completely bound the entire circular feature. However, this standard does not give any guidelines to establish this region. Many algorithms have been developed for roundness evaluation (Dowling et al., 1997). Among these algorithms, minimum-zone fitting and least-squares fitting are two commonly adopted approaches. It has been shown that unlike minimum-zone fitting, least-squares fitting does not yield a minimum roundness value from the inspection data (Lin et al., 1995; Murthy, 1986). To be consistent with the tolerance definition in the ASME standard, most algorithms have focused on obtaining the minimum roundness value from the inspection data.

Many researchers have cast the roundness evaluation as the solution of a general optimization problem (Choi and Kurfess, 1999; Gou et al., 1999; Sharma et al., 2000; Weber et al., 2002; Yau and Menq, 1996). To get a value that is close to the global minimum, some researchers addressed the initial conditions and adopted coordinate transformation techniques (Endrias and Feng, 2003; Lai and Chen, 1996) or suggested approximating orthogonal residuals by functions that are linear in the feature parameters (Shunmugam, 1991).

Other researchers based their algorithms on computational geometric techniques. Some adopted Voronoi diagrams (Huang, 1999; Novaski and Barczak, 1997; Samuel and Shunmugam, 2000; Roy and Zhang, 1992), convex hull or convex polygon techniques (Huang, 2001; Kaiser, 1998), or a data partition approach (Rajagopal and Anand, 1999). Moreover, some artificial intelligence techniques, such as neural networks, were also adopted for roundness evaluation (Suen and Chang, 1997). Chou et al. (2001) considered roundness evaluation in the 3D space as the measured points are impossible to be exactly on the same plane.

All the above algorithms are developed to evaluate the roundness of individual circular feature. So far, there is no concept reported in the literature that describes the overall quality status for a batch of circular features. For quality control, however, it is very important to know the overall quality for a batch of manufactured parts. Hence the concept that describes the worst possible case among a batch of circular features is very useful for the overall quality improvement of the batch.

This paper addresses the characterization and evaluation of batch roundness. The definition of batch roundness and its evaluation algorithm are given in the next section. Sections 3 and 4 present and discuss the implementation results in detail using simulated and experimental data sets, respectively. Section 5 wraps up this paper with concluding statements.

\section{BATCH ROUNDNESS}

\subsection{Definition}

The concept of batch roundness presented in this paper is defined as follows: 
Batch Roundness is the worst possible roundness value of a circular feature among a batch of the same circular features manufactured under the same conditions.

Such a batch of circular features has the following attributes: (1) the systematic form error component of every circular feature is very similar; and (2) the random form error component of every circular feature is also very similar. This means that the statistical parameters (mean $\mu_{r}$ and standard deviation $\sigma_{r}$ ) of the fitted residuals of every circular feature in the batch should be very close to each other.

Once the deterministic profile of a circular feature in the batch is determined, the standard deviation $\sigma_{r}$ of the fitted residuals is available. Then, an inner and an outer profile can be obtained by shifting the deterministic profile $3 \sigma_{r}$ at both sides (Fig. 1). The probability for every point on the deterministic profile to lie in between the inner and outer profiles is then 0.9974 . The probability for every point on the deterministic profile to lie within the tolerance zone, which is bounded by the two concentric circles in the figure, will be larger than 0.9974 . The gap between the two concentric circles that bound the inner and outer profiles thus represents the worst possible roundness value for the batch of circular features.

\subsection{Evaluation}

According to the above discussion, separating circular form errors into two components, systematic and random, is the key to obtain the deterministic profile from the discrete measurement data for batch roundness evaluation. In this paper, the objective function for characterizing the deterministic profile is defined as

$$
\operatorname{Min}_{x_{0}, y_{0}}\left(\sum_{i=1}^{N} e_{i}^{2}\right)
$$

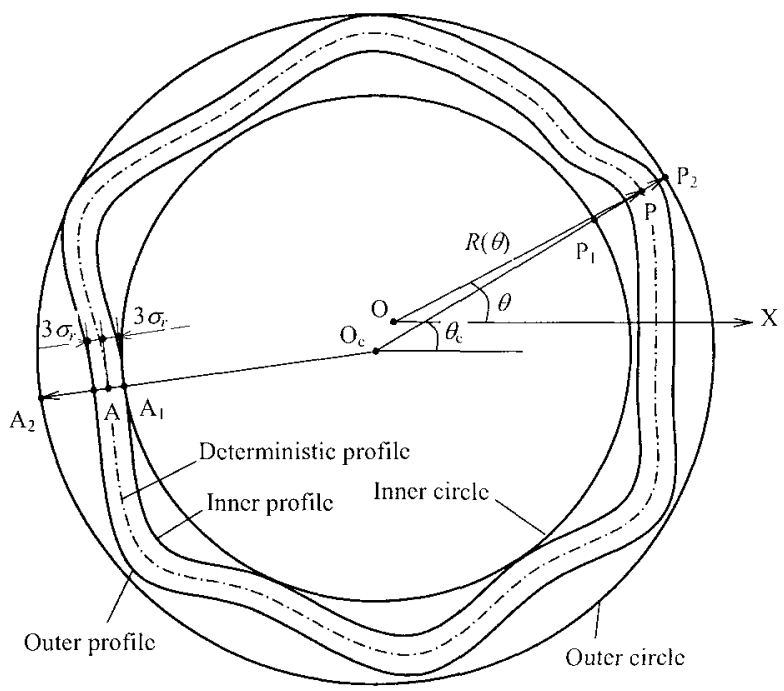

Figure 1 - Batch roundness evaluation. 
where $N$ is the number of the discrete data points, $e_{i}$ the fitted residual of the $i$ th data point $\left(e_{i}=R\left(\theta_{i}\right)-R_{i}\right) . R_{i}$ is the distance between the reference center $\mathrm{O}\left(x_{0}, y_{0}\right)$ and the $i$ th data point $\left(x_{i}, y_{i}\right)$ and it can be expressed as

$$
R_{i}=\sqrt{\left(x_{i}-x_{0}\right)^{2}+\left(y_{i}-y_{0}\right)^{2}}
$$

$R\left(\theta_{i}\right)$ is the function value of the deterministic profile $R(\theta)$ at $\theta_{i}$, and $R(\theta)$ is expressed as

$$
R(\theta)=R+S F C
$$

where $R=R_{0}+d R, R_{0}$ denotes the nominal radius of the circular feature and $d R$ the change in its radius. $d R$ is a constant and $S F C$ (sum of Fourier components) represents the sinusoidal fluctuation of the deterministic profile in the radial direction. $S F C$ can be expressed as

$$
S F C=\sum_{k=1}^{N_{d}} C_{l_{k}} \cos \left(l_{k} \theta+\phi_{l_{k}}\right)
$$

where $N_{d}$ is the number of Fourier components included in the deterministic profile; $C_{l_{k}}, l_{k}$ and $\phi_{l_{k}}$ are respectively the amplitude, frequency and phase angle of the $k$ th weighted Fourier component (Desta et al., 2003). To guarantee the correct characterization of the deterministic profile, a large sample size of 180 is adopted in the case studies in Sections 3 and 4 using both simulated and experimental data.

To calculate the Fourier components, it is necessary to determine the reference center. The initial coordinates of the reference center are

$$
\left\{\begin{array}{l}
x_{0}=\frac{1}{N} \sum_{i=1}^{N} x_{i} \\
y_{0}=\frac{1}{N} \sum_{i=1}^{N} y_{i}
\end{array}\right.
$$

Once the reference center is obtained, the Fourier components are calculated by Fast Fourier Transform (FFT). The procedure for characterizing the deterministic profile is an iterative process of solving the optimization problem expressed in Eq. (1). In this process, the Powell's method is applied as the optimization method.

After the deterministic profile is obtained, it can be shifted $3 \sigma_{i}$ at both sides to form an inner and an outer profile. Then, two concentric circles that contain the inner and outer profiles can be determined by the following optimization problem

$$
\underset{x_{i}, y_{i}}{\operatorname{Min}}\left(R_{\max }-R_{\min }\right)
$$

where $R_{\max }$ and $R_{\min }$ are respectively the longest and shortest distances from the center $\mathrm{O}_{c}\left(x_{c}, y_{c}\right)$ of the two concentric circles to the outer and inner profiles. The batch roundness is then given by the gap between the determined concentric circles.

\subsection{Profile Confidence Level}

Profile confidence level $P_{c l}$ indicates the probability for the characterized deterministic profile to lie in the region bounded by the two concentric circles. It is regarded as the average confidence level of every point on the deterministic profile. 
Once the center $\mathrm{O}_{c}\left(x_{c}, y_{c}\right)$ of the two concentric circles and their radii $R_{i n}$ and $R_{o u l}$ are determined, the profile confidence level can be calculated. For a point $P$ on the deterministic profile, the distances from $P$ to $P_{1}$ and $P_{2}$ can be determined and let them be $d_{1}$ and $d_{2}$, respectively (Fig. 1). Then the confidence level of point $P$ is

$$
\begin{aligned}
p & =\int_{-d_{j}^{\prime \prime}}^{r_{2}^{\prime}} \varphi(x) d x=\int_{-d_{1}^{\prime \prime}}^{0} \varphi(x) d x+\int_{0}^{r^{\prime}} \varphi(x) d x \\
& =\sum_{i=0}^{m_{1}} \frac{1}{2 n_{1}}\left[\varphi\left(x_{i}\right)+\varphi\left(x_{i+1}\right)\right]+\sum_{j=0}^{m^{2}} \frac{1}{2 n_{2}}\left[\varphi\left(x_{j}\right)+\varphi\left(x_{j+1}\right)\right]
\end{aligned}
$$

where $d_{1}{ }^{r}=d_{1} / \sigma_{r}, d_{2}{ }^{r}=d_{2} / \sigma_{r}, \sigma_{r}$ is the standard deviation of the fitted residuals, and $\varphi(x)=e^{-x^{2} / 2} / \sqrt{2 \pi}$. The profile confidence level can then be calculated as

$$
P_{c i}=\frac{1}{2 \pi} \int_{0}^{2 \pi} p d \theta=\frac{1}{2 \pi} \sum_{i=0}^{n} \frac{1}{2 n}\left(p_{i}+p_{i+1}\right)
$$

\section{SIMULATION STUDY}

The general equation for simulating a machined circular profile is given by

$$
\left\{\begin{array}{l}
x_{i}=\left(R+S F C+\varepsilon_{i}\right) \cos \theta_{i} \\
y_{i}=\left(R+S F C+\varepsilon_{i}\right) \sin \theta_{i}
\end{array} \quad(i=1,2, \Lambda, S)\right.
$$

where $R$ denotes the nominal radius, $S F C$ denotes the sum of Fourier components of the deterministic profile (which determines the systematic form error $S_{e}$ ), $\theta_{i}$ is the phase angle of the $i$ th data point, $S$ the sample size, and $\varepsilon_{i}$ a value from a set of normal deviations generated to simulate the random form error. Forty-five samples were produced to represent a batch of 45 circular features. The nominal radius in Eq. (9) was set to $15 \mathrm{~mm}$ and $S F C$ consisted of the following five components

$$
S F C=0.005 \cos \theta-0.007 \sin 2 \theta+0.015 \cos 4 \theta-0.02 \sin 7 \theta-0.012 \cos 10 \theta
$$

To investigate the effect of random errors, five cases have been analyzed by gradually increasing the random ratio. The results are listed in Table 1 . In the table, $3 \sigma / S_{e}$ denotes the random ratio, $\bar{r}_{d}$ the average individual roundness of the 45 simulated circular features, $V_{r_{d}}$ the roundness variation of the 45 individual roundness values, $\bar{R}_{d}$ the average of the batch roundness values evaluated from the 45 circular features (each circular feature gives an estimated batch roundness value), $V_{r_{d}}$ the batch roundness variation of the 45 batch roundness values, and $\bar{P}_{c /}$ the average profile confidence level.

When there is no random error $\varepsilon_{i}$, the resulting batch roundness variation is 0 . In this case, the characterized deterministic profiles of the 45 simulated circular features are exactly the same. This results in the systematic form error $S_{e}$ to be consistently $0.0824 \mathrm{~mm}$, which is also the batch roundness. The profile confidence level is $100 \%$. For the random ratio of 0.25 , the typical characterized deterministic profile of the simulated circular features is

$$
\begin{aligned}
R(\theta)= & 15.000011+0.039654 \cos (\theta+0.564780)+0.018833 \cos (7 \theta+1.562637) \\
& +0.015077 \cos (4 \theta-0.025804)-0.010998 \cos (10 \theta-0.079321) \\
& +0.007483 \cos (2 \theta+1.418556)+0.002648 \cos (47 \theta+0.424704)
\end{aligned}
$$


The calculation results show that the evaluated batch roundness fluctuates around $0.1242 \mathrm{~mm}$ with a variation of $0.0109 \mathrm{~mm}$. The profile confidence level is about $99.995 \%$ that is larger than $99.74 \%$. Comparatively, the individual roundness fluctuates intensively around $0.0969 \mathrm{~mm}$ with a large variation of $0.0201 \mathrm{~mm}$.

As the random ratio further increases, both individual roundness and batch roundness increase. Their variation and the difference between the two roundness values also increase, but the profile confidence level decreases very slightly. However, compared to individual roundness, the evaluated batch roundness values are more consistent which confirms that the batch roundness can be estimated from just one data set sampled from any individual circular feature in the batch.

Table 1 - Results using simulated data.

\begin{tabular}{|c|c|c|c|c|c|c|}
\hline $3 \sigma / S_{e}$ & $\bar{r}_{d}(\mathrm{~mm})$ & $V_{d d}(\mathrm{~mm})$ & $\bar{R}_{d}(\mathrm{~mm})$ & $V_{R_{d}}(\mathrm{~mm})$ & $\bar{P}_{c l}(\%)$ & $\bar{R}_{d}-\bar{r}_{d d}(\mathrm{~mm})$ \\
\hline 0 & 0.0822 & 0.0004 & 0.0824 & 0 & 100 & 0.0002 \\
\hline 0.25 & 0.0969 & 0.0201 & 0.1242 & 0.0109 & 99.995 & 0.0273 \\
\hline 0.5 & 0.1210 & 0.0342 & 0.1653 & 0.0145 & 99.994 & 0.0443 \\
\hline 0.75 & 0.1429 & 0.0411 & 0.2133 & 0.0212 & 99.993 & 0.0704 \\
\hline 1 & 0.1682 & 0.0585 & 0.2593 & 0.0272 & 99.992 & 0.0911 \\
\hline
\end{tabular}

\section{EXPERIMENTAL STUDY}

To demonstrate the presented concept and evaluation algorithm, 45 bosses with the same dimensions, $20 \mathrm{~mm}$ in diameter and $19 \mathrm{~mm}$ in height, were machined on one plate by the same end milling operation on a Fadal VMC 4020 vertical machining center. The workpiece material was 6061-T6 aluminum. The cutting tool was M42 HSS end mill with $6.35 \mathrm{~mm}$ cutting diameter, two right-handed flutes and $31.75 \mathrm{~mm}$ flute length. After the bosses were machined, a DEA Swift DCC coordinate measuring machine was used to measure these bosses in a single set-up. For each boss, 180 equidistant data points were taken at the depth of $10 \mathrm{~mm}$.

Although much effort was made to keep the manufacturing conditions the same during machining, it is impossible to make the manufacturing conditions exactly the same all the time. Besides, the measuring conditions cannot be kept exactly the same all the time, either. Therefore, both the systematic form errors and the random form errors will be different from boss to boss.

To judge whether a boss belongs to the same batch, a two-sigma criterion is introduced. For the systematic form errors of all 45 bosses, the mean $m_{1}$ is 0.0401 $\mathrm{mm}$ and the standard deviation $\sigma_{1}$ is $0.0047 \mathrm{~mm}$. The two-sigma criterion sets a range between $0.0307 \mathrm{~mm}$ and $0.0495 \mathrm{~mm}$. Since the obtained systematic form errors for bosses No. 20 and No. $31(0.0528 \mathrm{~mm}$ and $0.0302 \mathrm{~mm}$ respectively) fall outside the range, they are regarded as odd bosses and removed from the batch. Similarly, bosses No. 2 and No. 42 were removed due to their large standard deviations of the fitted residuals.

Figure 2 shows the evaluation results for the rest of 41 bosses. It can be seen that the batch roundness fluctuates around $0.0773 \mathrm{~mm}$ with a variation of $0.0151 \mathrm{~mm}$. Compared to the individual roundness, which fluctuates intensively around 0.0615 


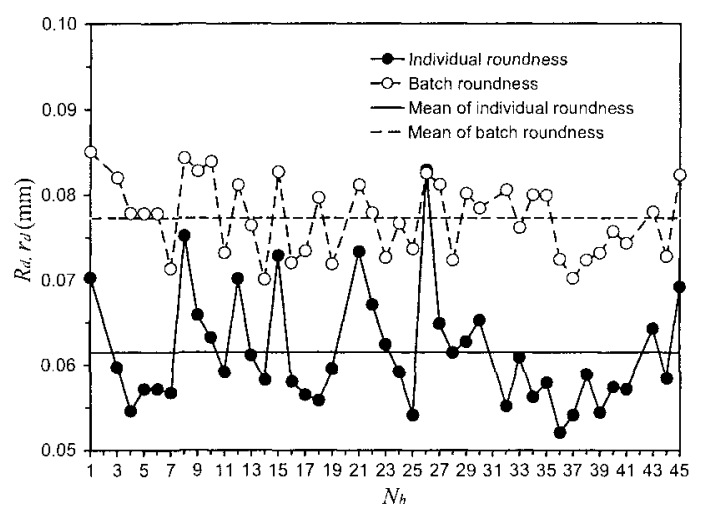

Figure 2-Batch roundness evaluation results.

$\mathrm{mm}$ with a variation of $0.0308 \mathrm{~mm}$, the evaluated batch roundness values are very consistent. Note that the individual roundness of boss No. 26 is a little larger than the corresponding batch roundness, $0.0825 \mathrm{~mm}$. This shows that forming the tolerance region by shifting $\pm 3 \sigma_{r}$ does not guarantee that this tolerance region covers all cases with the same statistical attributes. However, the profile confidence level shows that the average profile confidence level of the rest 40 bosses is $99.986 \%$. This value is indeed high enough to be considered satisfactory in practice.

\section{CONCLUSIONS}

For mechanical parts with circular features, the concept of batch roundness, which describes the worst possible case of roundness in a batch, helps indicate the overall quality status of the batch of mechanical parts. The simulation study shows that the evaluated batch roundness values are larger than the corresponding individual roundness values. The experimental work shows that in some cases the individual roundness may exceed the corresponding evaluated batch roundness. In general, however, the probability for the individual roundness to exceed the batch roundness is very small as the calculated profile confidence level is satisfactorily high.

Although the concept and the evaluation algorithm presented in this paper is used for evaluating the overall quality status for a batch of circular features, the basic principle is of general significance and can be applied to other types of geometric features. The reason is that every manufactured feature should have a deterministic profile. Once the deterministic profile of the interested feature is characterized, its batch tolerance region can be evaluated similarly.

\section{ACKNOWLEDGMENTS}

This research was in part supported by Natural Sciences and Engineering Research Council and The AUTO21 Network of Centres of Excellence of Canada. 


\section{REFERENCES}

1. Choi W, Kurfess TR. Dimensional measurement data analysis, part 2: minimum zone evaluation. ASME Journal of Manufacturing Science and Engineering 1999; 121:246-250.

2. Chou SY, Lin SW, Chen CH. Assessing circularity in three dimensions. ASME Journal of Manufacturing Science and Engineering 2001; 123: 128-134.

3. Desta MT, Feng HY, OuYang D. Characterization of general systematic form errors for circular features. International Journal of Machine Tools and Manufacture 2003; 43(11): 1069-1078.

4. Dowling MM, Griffin PM, Tsui KL, Zhou C. Statistical issues in geometric feature inspection using coordinate measuring machines. Technometrics 1997; 39(1): 3-24.

5. Endrias DH, Feng HY. Minimum-zone form tolerance evaluation using rigid-body coordinate transformation. ASME Journal of Computing and Information Science in Engineering 2003; 3(1): $31-38$.

6. Garavelli AC. Performance analysis of a batch production system with limited flexibility, International Journal of Production Economics 2001; 69(1): 39-48.

7. Gou JB, Chu YX, Li ZX. A geometric theory of form, profile, and orientation tolerances. Precision Engineering 1999; 23: 79-93.

8. Heywood MI, Chan M-C, Chatwin CR. Application of stochastic real-valued reinforcement neural networks to batch production rescheduling. Journal of Engineering Manufacture 1997; 211(8): 591603.

9. Huang J. A new strategy for circularity problems. Precision Engineering 2001; 25: 301-308.

10. Huang J. An exact solution for the roundness evaluation problems. Precision Engineering 1999; 23: $2-8$

11. Kaiser MJ, Generalized zone separation functions for convex perfect forms and incomplete data sets. International Journal of Machine Tools and Manufacture 1998; 38(4): 375-404.

12. Koziarski A, Krolikowski KJ. The calculation of costs of grinding on conventional and $\mathrm{CNC}$ grinding machines in small-batch shaft production. International Journal of Machine Tools and Manufacture $1998 ; 28(2): 123-130$.

13. Lai JY, Chen IH. Minimum zone evaluation of circles and cylinders. International Journal of Machine Tools and Manufacture 1996; 36(4): 435-451.

14. Lee $R$, Leonard $R$. Technical and financial considerations of $A G V$ use within multi-product and batch manufacture. Computer Integrated Manufacturing Systems 1992; 5(2): 97-104.

15. Lin SS, Varghese $\mathrm{P}$, Zhang C, Wang HPB. A comparative analysis of CMM form-fitting algorithms. ASME Mantufacturing Review 1995; 8(1): 47-58.

16. Murthy TSR. A comparison of different algorithms for circularity evaluation. Precision Engineering 1986; 8(1): 19-23.

17. Novaski O, Barczak ALC. Utilization of Voronoi diagrams for circularity algorithms. Precision Engineering 1997; 20(3): 188-195.

18. Primrose PL. Economics of batch manufacture. Computer Integrated Manufacturing Systems 1993; 6(2): 109-116.

19. Rajagopal K, Anand S. Assessment of circularity error using a selective data partition approach. International Journal of Production Research 1999; 37(17): 3959-3979.

20. Roy U, Zhang $X$. Establishment of a pair of concentric circles with the minimum radial separation for assessing roundness error. Computer-Aided Design 1992; 24(3): 161-168.

21. Samuel GL, Shunmugam MS. Evaluation of circularity from coordinate and form data using computational geometric techniques. Precision Engineering 2000; 24: 251-263.

22. Sharma R, Rajagopal K, Anand S. A genetic algorithm based approach for robust evaluation of form tolerances. Journal of Manufacturing Systems 2000;19(1): 46-57.

23. Shiu Y-C. An economic batch production quantity model with learning curve-dependent effects: a technical note. International Journal of Production Economics 1991; 25: 35-38.

24. Shunmugam MS. Criteria for computer-aided form evaluation. ASME Journal of Engineering for Industry 1991; 113: 233-238.

25. Suen DS, Chang CN. Application of neural network interval regression method for minimum zone circle roundness. Measurement Science and Technology 1997; 8(3): 245-252.

26. Weber T, Motavalli S, Fallahi B, Cheraghi SH. A unified approach to form error evaluation. Precision Engineering 2002; 26(3): 269-278.

27. Yau HT, Menq CH. A unified least-squares approach to the evaluation of geometric errors using discrete measurement data. International Journal of Machine Tools and Manufacture 1996; 36(11): $1269-1290$. 